\title{
Diagnose und Management der Osteoporose bei Diabetes mellitus (Update 2019)
}

\author{
Gemeinsame Leitlinie der Österreichischen Gesellschaft für Knochen- und \\ Mineralstoffwechsel und der Österreichischen Diabetes Gesellschaft
}

Christian Muschitz · Alexandra Kautzky-Willer · Martina Rauner · Yvonne Winhöfer-Stöckl · Judith Haschka

Online publiziert: 12. April 2019

(C) Der/die Autor(en) 2019

\begin{abstract}
Zusammenfassung Diabetes mellitus und Osteoporose zählen zu den häufigsten chronischen Erkrankungen und kommen deshalb beide häufig in ein und demselben Individuum vor. Da die Prävalenz beider mit steigendem Alter zunimmt, wird in Anbetracht der Altersstruktur unserer Bevölkerung deren Häufigkeit zunehmen.

Patienten mit Diabetes haben ein erhöhtes Risiko für Fragilitätsfrakturen. Die Pathophysiologie ist unklar und vermutlich multifaktoriell.

Longitudinale Studien haben den Nachweis erbracht, dass das Fracture Risk Assessment Tool (FRAX) und die Knochendichte (BMD) mittels DXA (T-score) Messungen und einem eventuell vorhandenen Trabecular Bone Score (TBS) das individuelle Frakturrisiko vorhersagen können. Hierfür muss allerdings eine Adjustierung vorgenommen werden, um das Risiko nicht zu unterschätzen.

Es gibt derzeit aus osteologischer Sicht noch nicht den optimalen Ansatz, da es keine Studien mit rein diabetischen Patienten und Osteoporose gibt.
\end{abstract}

Priv.-Doz. Dr. C. Muschitz $(\bowtie) \cdot$ J. Haschka

Externe Lehre, Medizinische Universität Wien, Wien,

Österreich

christian.muschitz@meduniwien.ac.at

Priv.-Doz. Dr. C. Muschitz

II. Medizinische Abteilung, Krankenhaus der Barmherzigen

Schwestern Wien, Stumpergasse 13, 1060 Wien, Österreich

A. Kautzky-Willer · Y. Winhöfer-Stöckl

Gender Medicine Unit, Klinische Abteilung für

Endokrinologie und Stoffwechsel, Universitätsklinik für

Innere Medizin III, Medizinische Universität Wien, Wien,

Österreich

M. Rauner

Bone Lab Dresden, Medizinische Klinik und Poliklinik III,

Medizinische Fakultät, Technische Universität Dresden,

Dresden, Deutschland
Patienten mit Diabetes mellitus und einem erhöhten Frakturrisiko sollten genauso wie Patienten ohne Diabetes und einem erhöhten Frakturrisiko behandelt werden.

Der Vitamin-D-Spiegel sollte auf jeden Fall immer optimiert werden und auf eine ausreichende Kalziumaufnahme (vorzugsweise durch die Nahrung) ist zu achten.

Bei der Wahl der antihyperglykämischen Therapie sollten Substanzen mit nachgewiesen negativem Effekt auf den Knochen weggelassen werden. Bei Vorliegen einer Fragilitätsfraktur ist auf jeden Fall - unabhängig von allen vorliegenden Befunden - eine langfristige spezifische osteologische Therapie indiziert.

Zur Prävention von Fragilitätsfrakturen sind antiresorptive Medikamente die erste Wahl, entsprechend der nationalen Erstattungskriterien auch anabole Medikamente. Das Therapiemonitoring soll im Einklang mit der nationalen Osteoporose Leitlinie erfolgen.

Schlüsselwörter Diabetes · Osteoporose · Frakturen · Frakturrisiko $\cdot$ Medikation

Diagnosis and management of patients with diabetes and co-existing osteoporosis (Update 2019)

Common guideline of the Autrian Society for Bone and Mineral Research and the Austrian Diabetes Society

Summary Fragility fractures are increasingly recognized as a complication of both type 1 and type 2 diabetes, with fracture risk that increases with disease duration and poor glycemic control. The identification and management of fracture risk in these patients remains challenging. This manuscript explores 
the clinical characteristics of bone fragility in adults with diabetes and highlights recent studies that have evaluated areal bone mineral density (BMD), bone microstructure and material properties, biochemical markers, and fracture prediction algorithms (FRAX) in these patients. It further reviews the impact of diabetes drugs on bone tissue as well as the efficacy of osteoporosis treatments in this population. An algorithm for the identification and management of diabetic patients at increased fracture risk is proposed.

Keywords Diabetes - Diabetes-related bone disease Fracture $\cdot$ Osteoporosis · Medication

\section{Epidemiologie des Diabetes mellitus und osteo- porotische Fragilitäts-Frakturen}

Diabetes mellitus und Osteoporose zählen zu den häufigsten chronischen Erkrankungen und kommen deshalb beide häufig in ein und demselben Individuum vor, weshalb davon ausgegangen wird, dass sie in Zusammenhang stehen. Da die Prävalenz beider mit steigendem Alter zunimmt, wird in Anbetracht der Altersstruktur unserer Bevölkerung deren Häufigkeit zunehmen.

Rezente Metaanalysen mit rund 140.000 Patientinnen und Patienten mit Typ 1 Diabetes (T1DM) zeigen ein gepooltes relatives Risiko (RR) für eine Fraktur von 3,16 , für eine Hüftfraktur von 3,78 und für eine vertebrale Fraktur von 2,88. Das RR einer hüftgelenksnahen Fraktur bei Frauen mit T1DM im Vergleich zu Frauen ohne Diabetes beträgt 5,19 [1]. Das Frakturrisiko steigt mit zunehmendem Lebensalter an, speziell Hüftfrakturen treten bei T1DM etwa 10-15 Jahre früher auf [2].

Bei Typ 2 Diabetes (T2DM) weisen Populations-basierte Daten von rund 33.000 Patientinnen und Patienten den T2DM als stärksten Prädiktor für niedrigtraumatische (=osteoporotische) Frakturen bei Männern (RR 2,38) und bei Frauen (RR 1,87) aus [3]. Mit einer durchschnittlichen Odds-Ratio (OR) für Frakturen von 1,5 ist der T2DM nur für rund $4 \%$ aller osteoporotischen Frakturen ursächlich in Zusammenhang zu bringen. Dem gegenüber ist allerdings die global steigende Inzidenz von Patientinnen und Patienten mit T2DM (rund 425 Mio. sowie rund 320 Mio. mit einer gestörten Glucose-Toleranz) gegenüber zu stellen. Zusätzlich zu diesem direkten Risiko kommen noch weitere klinische Risikofaktoren (clinical risk factors, CRF), die mit einem Diabetes einhergehen (z. B. multiple Stürze, Neuro- und Retinopathie, etc.) und das individuelle Frakturrisiko zusätzlich erhöhen (Tab. 2; [4]).

\section{Diabetes assoziierte Risikofaktoren für Frakturen}

Diabetes per se ist ein klinischer Risikofaktor für ein erhöhtes Frakturrisiko. Bei T2DM spielen das Alter und die Dauer des Diabetes eine wichtige Rolle. Sowohl bei Frauen als auch bei Männern >40 Jahren ist T2DM ein unabhängiger Risikofaktor für sämtliche osteoporotische Frakturen (Hazard Ratio, HR 1,32). Das Alter beeinflusst das Risiko dahin gehend, dass jüngere Patienten ein höheres Risiko für Hüftfrakturen haben (HR Alter <60 Jahre: 4,67; HR Alter 60-69 Jahre: 2,68; HR Alter 70-79 Jahre: 1,57; HR Alter >80 Jahre: 1,42) [5]. Entscheidend ist außerdem die Dauer der Erkrankung. In den ersten fünf Jahren der Erkrankung kommt es zu keiner Erhöhung des relativen Risikos (ein protektiver Effekt vermehrter Fettmasse wird diskutiert), das Risiko folgt allerdings einem biphasischem Verlauf mit einem zweiten Gipfel jenseits von zehn Jahren Erkrankungsdauer (HR 1,47) [6].

Die glykämische Kontrolle ist wichtig für die Beurteilung des individuellen Frakturrisikos. Ein HbAlc $>7 \%$ führt zu einem raschen Anstieg des Risikos mit einer erhöhten Mortalität nach Frakturen [7]. Zusätzlich hat eine schlechte glykämische Kontrolle einen negativen Einfluss auf die Mikroarchitektur des Knochens mit mikrovaskulären Komplikationen in diesem Organsystem [8].

\section{Einfluss der Behandlung des Diabetes auf das Frakturrisiko}

Das Verhältnis zwischen Diabetes und Knochenfragilität und die Identifizierung jener Patientinnen und Patienten mit einem erhöhten Risiko für Frakturen wird zusätzlich durch die Eigenschaften antidiabetogener

Tab. 1 Einfluss antidiabetogener Medikamente auf BMD (bone mineral density, Knochendichte) und Frakturrisiko in T2DM

\begin{tabular}{|c|c|c|}
\hline Antidiabetogene Medikation & BMD & Frakturrisiko \\
\hline Metformin & $\leftrightarrow / \uparrow$ & $\downarrow / \leftrightarrow$ \\
\hline Sulfonylharnstoffe & $\mathrm{KD}$ & $\leftrightarrow / \uparrow$ \\
\hline Thiazolidinedione & $\downarrow \downarrow / \leftrightarrow$ & $\uparrow \uparrow / \leftrightarrow$ \\
\hline Insulin & $\leftrightarrow$ & $\uparrow$ \\
\hline \multicolumn{3}{|l|}{ Inkretin Mimetika } \\
\hline DPP4 Inhibitoren & $\leftrightarrow$ & $\downarrow / \leftrightarrow$ \\
\hline GLP1 Analoga & $\uparrow / \leftrightarrow$ & $\leftrightarrow$ \\
\hline SGLT2 Inhibitoren & $\leftrightarrow$ & $\leftrightarrow$ \\
\hline Canagliflozin & $\leftrightarrow$ & $\uparrow(?)$ \\
\hline
\end{tabular}


Tab. 2 Effekte spezifischer Osteoporose Medikamente bei Patienten mit einem T2DM auf BMD und Frakturrisiko [45]

\begin{tabular}{l|l|l|}
\hline Spezifisches 0steoporose Medikament & BMD (T2DM) & Frakturrisiko in Bezug auf T2DM \\
\hline Alendronat & $\uparrow$ & $\mathrm{KD} / \leftrightarrow$ \\
\hline Risedronat & $\uparrow$ & $\mathrm{KD}$ \\
\hline Ibandronat & $\mathrm{KD}$ & $\mathrm{KD}$ \\
\hline Zoledronat & $\mathrm{KD}$ & $\mathrm{KD}$ \\
\hline Raloxifen & $\mathrm{KD}$ & $\downarrow / \leftrightarrow$ \\
\hline Denosumab & $\mathrm{KD}$ & $\mathrm{KD}$ \\
\hline Teriparatid & $\uparrow$ & $\leftrightarrow$ \\
\hline $\begin{array}{l}\uparrow \text { Verbesserung/Erhöhung, } \downarrow \text { Verminderung, } \leftrightarrow \text { unverändert } \\
K D \text { keine Daten }\end{array}$ & \\
\hline
\end{tabular}

Medikamente auf das Skelett beeinflusst (Tab. 1). Obwohl es keine einzige prospektive Studie mit einem primären Studienziel in Bezug auf Therapie des Diabetes und Knochenfragilität gibt, zeigen Daten aus epidemiologischen und Beobachtungsstudien ein heterogenes Muster von teilweise positiven, aber auch negativen Effekten auf den Knochenstoffwechsel.

\section{Lebensstilfaktoren}

Eine Veränderung des Lebensstils ist - nicht nur bei der Erkrankung Diabetes - eine der Säulen der nicht-medikamentösen Therapie. Grundsätzlich ist ein Gewichtsverlust, sofern keine Gegenmaßnahmen gesetzt werden, immer mit dem Verlust von Muskelund Knochenmasse verbunden. Sarkopenie und sarkopene Adipositas sind Risikofaktoren für Stürze und Gebrechlichkeit, daher ist immer auf eine ausreichende alimentäre Zufuhr von Proteinen und progressives Widerstandstraining zu achten.

Körperliche Aktivität während einer gezielten Gewichtsabnahme verbessert die Lebensqualität und senkt gleichzeitig zirkulierende Sclerostin-Spiegel, unabhängig vom Alter der Patienten [9].

Andere nicht-pharmakologische Maßnahmen sind - wie bei vielen anderen Erkrankungen - die Vermeidung von Nikotin und übermäßigem Alkoholgenuss.

Ein Vitamin D Mangel ist sowohl beim T1DM als auch beim T2DM mit hoher Prävalenz vorhanden. Obwohl der direkte Beweis für die Wirksamkeit eines optimierten Vitamin D Spiegels bei Adipositas bzw. Diabetes und/oder Insulin-Resistenz noch nicht in Studien als primärer Endpunkt nachgewiesen wurde, ist doch davon auszugehen, dass Patienten mit Diabetes ähnlich wie nicht-diabetische Kollektive davon profitieren. Ein adäquater Vitamin D Spiegel und eine suffiziente Aufnahme von Calcium (vorzugsweise über die Nahrung) sind daher eine Grundvoraussetzung - auch im Hinblick auf die Prävention eines sekundären Hyperparathyreoidismus. Möglicherweise sind anfänglich höhere Einzeldosen von Cholecalciferol notwendig, um einen suffizienten Spiegel zu erreichen [10]. Eine Supplementation hat allerdings keinen Schutz vor Frakturen, Sturz oder klinisch relevante Effekte auf die Knochendichte gezeigt [11].

\section{Glykämische Kontrolle}

Bei Patienten mit Diabetes besteht zusätzlich eine Fallneigung, welche wahrscheinlich zum erhöhten Frakturrisiko beiträgt. Die periphere Neuropathie, die Retinopathie mit Visusverschlechterung, vermehrte Stürze in der Anamnese, die Tendenz zu hypoglykämen Episoden, die Hypo- oder Hypertension bzw. die autonome Neuropathie sind hier beispielhaft $\mathrm{zu}$ nennen.

Eine eng eingestellte glykämische Kontrolle (HbAlc $<7 \%$ ) verringert das Frakturrisiko bei Diabetes, vor allem bei älteren Patienten. Allerdings ist sowohl die Hypoglykämie als auch die Hyperglykämie mit einem erhöhten Risiko für Fragilitätsfrakturen assoziiert, wahrscheinlich durch unterschiedliche Mechanismen [12]. Vor allem bei älteren Patienten mit Diabetes wird daher - um das Risiko für hypoglykäme Episoden zu vermeiden - eine weniger stringente Einstellung des Diabetes empfohlen, um das Sturzrisiko zu senken (EASD/ADA Guidelines) [13].

\section{Effekte antihyperglykämischer Therapie auf den Knochen}

\section{Metformin}

In vitro Studien zeigen einen positiven Effekt von Metformin auf die RUNX2 (Runt-related transcription factor 2) Expression mit einer vermehrten Aktivierung von Osteoblasten. In Mäusen und Ratten führt die Verabreichung von Metformin zu einer verbesserten Frakturheilung. Zudem kann es dem Knochenverlust in Modellen der Östrogendefizienz bzw. einer ketogenen Diät entgegenwirken. Klinische Daten im humanen und Tiermodell bestätigen einen neutralen bis positiven osteogenen Effekt in Bezug auf Frakturen und bei ketogener Ernährung, sodass diese Medikation in Bezug auf die Knochenqualität als sicher zu werten ist [14-16].

\section{Sulfonylharnstoffe}

Obwohl in vitro Untersuchungen keinen direkten Effekt von Sulfonylharnstoffen auf das Organsystem Knochen nachweisen konnten, zeigen epidemiologische Daten ein möglicherweise erhöhtes Frakturrisiko 
unter dieser Medikation. Daten zu Veränderungen der Knochendichte liegen nicht vor. Die Hypothese ist ein indirekt erhöhtes Sturzrisiko bedingt durch Hypoglykämien in dieser Substanzgruppe [14]. Hingegen zeigte sich ein neutraler Effekt auf die BMD in kleinen Patientenkollektiven [17, 18].

\section{Thiazolidinedione}

Sowohl in vitro als auch klinische Studien haben bei Rosiglitazon und Pioglitazon einen Verlust von Knochenstruktur nachgewiesen. Thiazolidinedione interagieren mit dem peroxisome proliferator-activated receptor (PPAR) $\gamma$, was zu einer Dysbalance zugunsten der Differenzierung von Adipozyten und zu Lasten der Differenzierung von Osteoblasten führt. Die klinische Konsequenz sind Frakturen. Eine rezente Metaanalyse bestätigt den negativen Einfluss von Pioglitazon beim weiblichen Geschlecht, aber auch für das männliche Geschlecht liegen ähnliche Ergebnisse vor [19]. Es wird daher nach derzeitigem Wissenstand empfohlen, bei postmenopausalen Frauen und auch bei Männern mit einem Risikoprofil für Fragilitätsfrakturen diese Substanzgruppe eher nicht einzusetzen [20, $21]$.

\section{Insulin}

In Observationsstudien wurde häufig ein erhöhtes Frakturrisiko für Patienten unter dieser Therapie beschrieben. Patientinnen und Patienten unter einer Insulin-Therapie bzw. unter einer Therapie mit Insulin Sekretagoga haben indirekt durch die Hypoglykämieinduzierten Stürze ein erhöhtes Risiko. Ein weiterer Grund dürfte die längere Krankheitsdauer ( $>5$ Jahre) und/oder eine schlechte glykämische Kontrolle zusammen mit sekundären Komplikationen (Retinopathie, Neuropathie, Nephropathie, etc.) sein [22].

\section{Inkretinmimetika}

Beide Inkretinmimetika Dipeptidyl Peptidase-4 (DPP4) Inhibitoren und Glucagon-like peptide-1 (GLP-1) Analoga haben beim T2DM ein mehrheitlich neutrales skeletales Sicherheitsprofil. Der wahrscheinlichste Mechanismus ist der neutrale bis positive Effekt von GLP-1 auf die Knochenformation durch Änderung der miRNA-Expression und das geringe Risiko in Bezug auf iatrogene Hypoglykämie [23].

\section{SGLT-2-Inhibitoren}

Rezente Daten über den Natrium-Glucose-Cotransporter 2 (SGLT2) Inhibitor Canagliflozin beschreiben einen negativen Effekt im Sinne einer Abnahme der Knochenmineraldichte (bone mineral density, BMD) und einer Zunahme des Knochenbruchrisikos. Der zugrunde liegende Mechanismus ist noch nicht vollständig entschlüsselt, aber SGLT-2-Inhibitoren hemmen im proximalen Tubulus die Reabsorption von Glucose und führen gleichzeitig zu einer vermehrten Reabsorption von Phosphat. Dies führt konsekutiv zu erhöhten Spiegeln von Serum Phosphat, was ein möglicher Trigger für Parathormon (PTH) und einen erhöhten Knochenstoffwechsel ist.

Konträr zu Canagliflozin gibt es derzeit keinen Hinweis auf einen negativen Einfluss von Empagliflozin oder Dapagliflozin auf das Organsystem Knochen, weshalb den beiden letztgenannten Substanzen der Vorzug in dieser Substanzklasse gegeben werden sollte. Allerdings konnte eine Metaanalyse von 20 Studien mit Dapagliflozin, Empagliflozin oder Canagliflozin keine eindeutige Erhöhung der Frakturraten nachweisen. Aus derzeitiger Sicht ist bei Patienten mit T2DM unter Canaglifozin möglicherweise das Frakturrisiko erhöht [24].

\section{Diagnostik}

\section{DXA-Knochendichtemessung}

Die Knochendichtemessung mittels DXA (dual energy x-ray absorptiometry) ist nach wie vor der Goldstandard. Die Definition einer Osteoporose von einem T-score $\leq-2,5$ basiert auf einer Definition der WHO aus dem Jahr 1994 und definiert die Erkrankung, jedoch nicht die individuelle Interventionsschwelle [25].

Die Mehrzahl der Studien bei Patienten mit T1DM zeigen, dass die BMD bei dieser Patientenpopulation deutlich vermindert ist [26]. Aufgrund der meist vorherrschenden Adipositas als Risikofaktor bei T2DM wäre grundsätzlich davon auszugehen, dass ein hoher Body Mass Index (BMI) und eine hohe BMD positiv miteinander korrelieren. Daher haben Patienten mit einem T2DM in der Regel eine 5-10\% höhere BMD im Vergleich zur nicht-diabetischen gesunden Population. Die höhere BMD ist vor allem beim jüngeren männlichen Geschlecht vorherrschend - interessanter Weise auch bei höheren HbAlc Werten. Die höhere BMD ist vor allem am Gewichts-tragenden Knochen zu sehen, jedoch nicht am Radius.

Die relativ höhere BMD bei T2DM schützt die Patienten jedoch nicht vor Frakturen. Die Mehrzahl der Patienten mit Frakturen haben einen T-score im osteopenen Bereich, also einem T-score $>-2,5$ [27]. Bei Frauen mit T2DM ist das individuelle Frakturrisiko im Gegensatz zu Frauen ohne Diabetes in etwa 0,5 Tscores als Korrekturfaktor tiefer als der tatsächliche Messwert anzusetzen (Abb. 1). Obwohl zahlreiche Studien in dieser Patientenpopulation bestätigen, dass die DXA-Messung systematisch das Frakturrisiko unterschätzt, werden unter Berücksichtigung dieses Korrekturfaktors vor allem ältere Patientinnen und Patienten adäquat stratifiziert [28].

Manche Studien bestätigen einen schnelleren Verlust an BMD auch an Gewichts-tragenden Knochen (z. B. Hüfte) unter T2DM als möglichen Grund für die erhöhte Frakturrate [29]. 


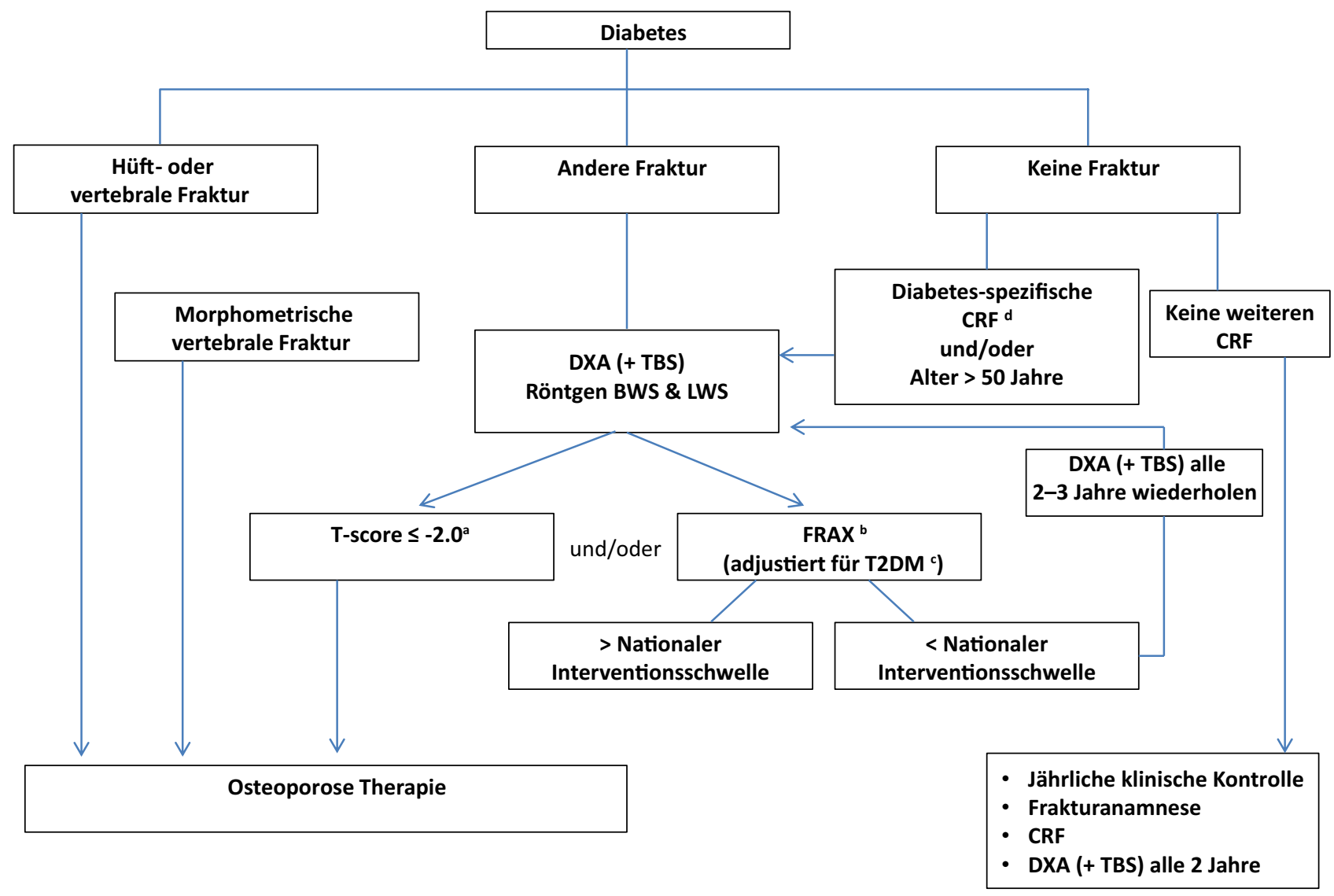

Abb. 1 Evaluation des Frakturrisikos bei Patienten mit Diabetes. ( ${ }^{a} B e i$ Diabetes ist das Frakturrisiko bei einem T-score $\leq-2,0$ gleich hoch wie beim nicht-diabetischen Patienten mit einem T-score $\leq-2,5$. bIn Österreich gilt folgende nationale Interventionsschwelle im FRAX: 10-Jahreswahrscheinlichkeit für

Mit dem Trabecular Bone Score (TBS) steht eine Methode zur Verfügung, um aus einer zweidimensionalen DXA-Untersuchung Informationen über die Knochenmikrostruktur der Lendenwirbelsäule zu generieren. Diese einheitslose Zahl spiegelt anhand der Analyse von Grauwert-Variogrammen der radiologischen Messung der Lendenwirbelkörper L1-L4 mit einer hohen Korrelation die trabekuläre Mikroarchitektur bei Osteoporose unabhängig von der BMD wider [30]. Der TBS-Software kann direkt im Rahmen der Messung mit, oder auch retrospektiv den Score berechnen, wodurch sich keine zusätzliche Strahlenbelastung für den Patienten ergibt. Im Gegensatz zur DXA-Methode ist der TBS bei Patienten mit T2DM tiefer als in einer nicht-diabetischen Population. In einer großen Populations-basierten Untersuchung wurden Patienten mit einem TBS von $<1230$ als Risikopatienten für Osteoporose-assoziierte Frakturen eingestuft, bei einem TBS von 1230-1310 ein mittleres Risiko. Bei T2DM fanden sich in Studien TBS Werte zwischen 1100 und 1200 [31].

Der TBS ist bei Patienten mit guter glykämischer Kontrolle höher und tiefer bei einem schlecht eingestellten T2DM. Der TBS ist somit ein unabhängiger major osteoporotic fracture: $\geq 20 \%$, hip fracture: $\geq 5 \%$. ${ }^{c} z$. B. mit TBS Korrektur oder der CRF „Rheumatoide Arthritis“ wird auf „Ja“ gesetzt. 'Zu diesen Frakturen zählen: Humerusfraktur, Schambeinastfraktur, Clavicula, Rippen (=nicht-vertebrale, nicht-Hüft Frakturen))

Prädiktor für das Frakturrisiko bei Diabetes (HR 1,27) bzw. auch ohne Diabetes (HR 1,31) [32].

Alternative Methoden wie etwa der Ultraschall am Calcaneus oder am Radius zeigen inkonklusive Ergebnisse bei T2DM [33].

\section{Mikroarchitektur und Knochenqualität}

Die BMD alleine - vor allem beim T2DM - erklärt nicht die erhöhte skeletale Fragilität. Sowohl in MRTUntersuchungen als auch mittels HR-pQCT (high resolution peripheral quantitative computed tomography) am Radius (nicht Gewichts-tragender Knochen) und an der Tibia (Gewichts-tragender Knochen) zeigt sich beim T2DM eine verschlechterte Mikroarchitektur. Die Trabekel beim T2DM sind im Vergleich zum nicht-diabetischen Patienten eher hypertrophiert. Im trabekulären Netzwerk finden sich allerdings auch größere Löcher, zusätzlich ist die kortikale Porosität (bis zu 16\%) gegenüber Patienten ohne T2DM erhöht. Die strukturelle Alteration mit hoher Heterogenität ist an der endokortikalen Übergangszone besonders ausgeprägt („Trabekularisierung der Kortikalis“). Zusätzlich gibt es einen geschlechtsspezifischen Unterschied 


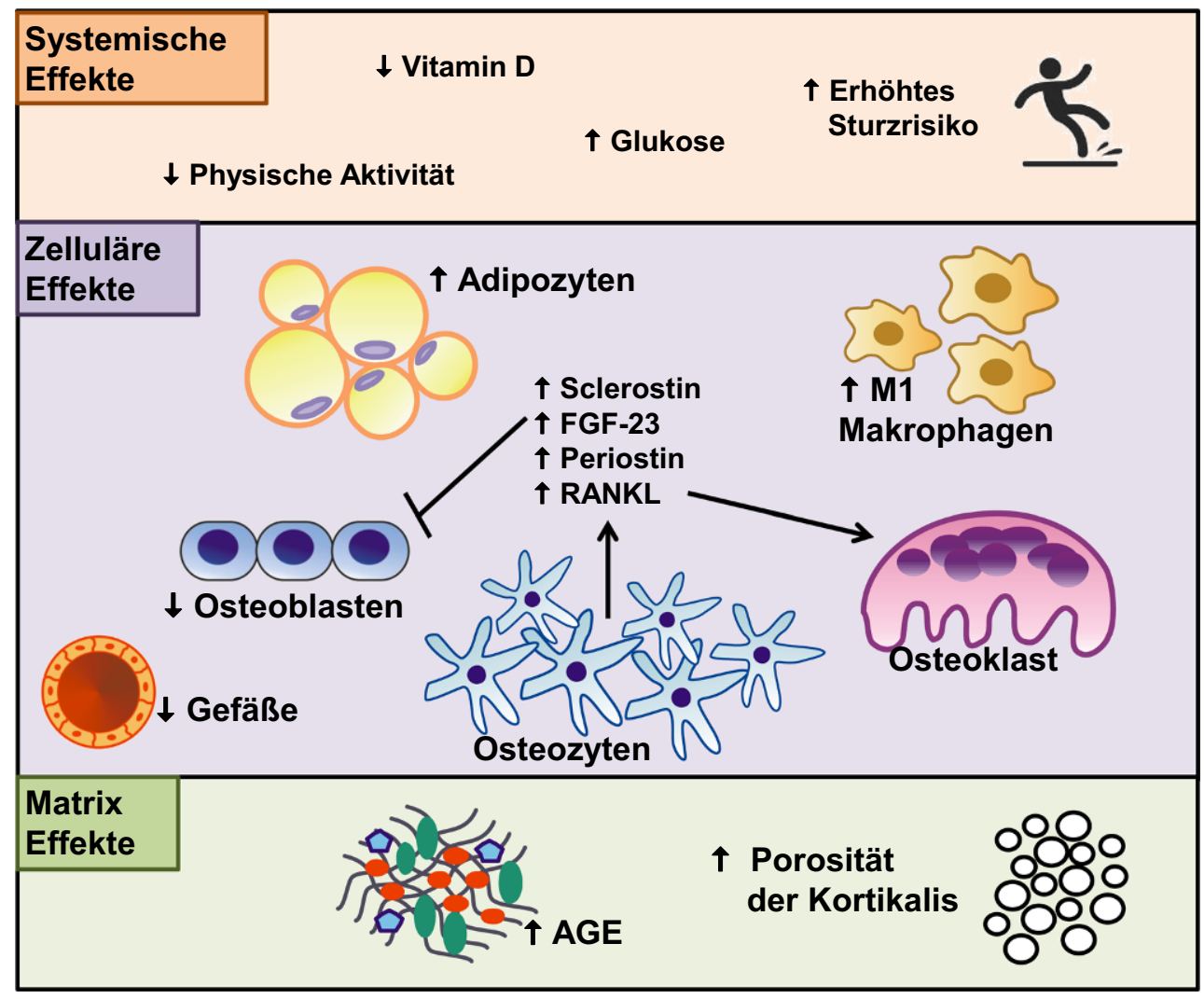

Abb. 2 Effekte des T2DM auf den Knochenstoffwechsel. Auf systemischer Ebene ist T2DM mit einem niedrigen Vitamin DSpiegel, hoher Glukosewerte, sowie eingeschränkter physischer Aktivität und einen erhöhten Sturzrisiko verbunden, alle, welche zu einer erhöhten Frakturrate führen. Auf zellulärer Ebene ist vor allem die Anzahl der Adipozyten erhöht, wobei die Anzahl der Osteoblasten sowie die Anzahl und Funktion der Gefäße vermindert sind. Osteozyten werden zur vermehrten Produktion von Sclerostin, Periostin, FGF-23 und RAN$\mathrm{KL}$ animiert, welche die Osteoblastendifferenzierung hemmen

mit schlechteren Werten beim weiblichen Geschlecht $[34,35]$.

Aufgrund dieser strukturellen Defizite bei T2DM ist die Knochenfestigkeit sowie die Steifigkeit und die Elastizität des Knochens in virtuellen FEA (finite element analysis) Untersuchungen vermindert. Microindentations-Untersuchungen zeigen zusätzlich strukturelle Einschränkungen als Ausdruck veränderter Kollagenverlinkungen in der Knochenmatrix aufgrund vermehrter AGEs (advanced gylcation endproducts). Diese Verlinkungen führen dazu, dass der Knochen an Elastizität und Flexibilität verliert. In Summe haben diese Untersuchungsergebnisse den Begriff der „Diabetoporose“ geprägt ([36]; Abb. 2).

\section{Knochenstoffwechsel: Histomorphometrie und Serum Marker}

Der Goldstandard zur Untersuchung des lokalen Knochenstoffwechsels ist die Histomorphometrie aus bioptischen Proben. Zur Abschätzung der tatsächlichen Aktivität ist einer der wichtigen Parameter die For- und die Osteoklastengeneration fördern. Zusätzlich fördern pro-inflammatorische M1-Makrophagen die Osteoklastengeneration und hemmen Osteoblasten. T2DM hat auch direkte Effekte auf die Knochenmatrix. So werden vermehrt advanced glycation endproducts (AGE) in die Kollagenmatrix eingebaut, die so zur Versteifung der Matrix beiträgt. Außerdem führt T2DM zur einer poröseren kortikalen Knochenstruktur, welches die mechanische Stabilität des Knochens beeinträchtigt

mationsrate bezogen auf eine Referenzoberfläche der Biopsie (BFR/BS, bone formation rate/bone surface). Bei diabetischen Patientinnen und Patienten ist dieser Parameter im trabekulären, endokortikalen und intrakortikalen Bereich um bis zu 70-80\% vermindert [37].

In der Mehrzahl der Studien wurde bei Patienten mit T2DM eine verminderte Aktivität von serologischen Formationsmarkern (procollagen type I N-terminal propeptide, PINP; osteocalcin OC) und Resorptionsmarkern (C telopeptide, CTX; tartrate-resistant acid phosphatase 5, TRAP5b) nachgewiesen [14]. Der Zusammenhang zwischen dem low bone turnover und der gleichzeitig nachgewiesenen strukturellen Alteration (kortikale Porosität) ist zum gegenwärtigen Zeitpunkt unklar.

\section{Alternative (experimentelle) biochemische Mar- ker der Knochenfragilität}

Bei Diabetes ist in der Knochenmatrix der Gehalt von Pentosidin, dem am häufigsten vorhandenen AGE, im Vergleich zu nicht-diabetischen Menschen deut- 
lich erhöht. Erhöhte Pentosidin-Spiegel im Knochen und im Serum korrelieren negativ mit der biomechanischen Stärke des Knochens. Untersuchungen bestätigen erhöhte Werte von AGEs und auch von sRAGE (soluble receptors for advanced glycation endproducts) als prädiktiven Faktor für eine erhöhte Inzidenz von klinischen und vertebralen Frakturen unabhängig von der BMD [38].

Sclerostin, der endogene Inhibitor des Wnt/ $\beta$-Catenin Signalweges und somit der Knochenformation durch Osteoblasten, ist bei T2DM deutlich erhöht. Die Höhe der Sclerostin-Spiegel korreliert bei diesen Patienten mit der Inzidenz von Fragilitätsfrakturen [39]. Bei T1DM verhalten sich die Sclerostin-Spiegel genau gegenläufig zur Inzidenz von Frakturen. Patienten im obersten Drittel der gemessenen Spiegel hatten ein um $81 \%$ geringeres Frakturrisiko verglichen mit Patienten mit Spiegeln im untersten Drittel [40]. Ob nun eine Erhöhung zirkulierender Sclerostin-Spiegel direkt die Dysfunktion von Osteozyten widerspiegelt und/ oder ein Marker für eine zusätzliche Angiopathie sind, bleibt derzeit noch unbeantwortet [41].

Serum Periostin bzw. dessen Fragmente sind mit einem erhöhten Frakturrisiko bei nicht-diabetischen Patienten vergesellschaftet. Derzeit laufen Studien in großen diabetischen Populationen zur Evaluation dieses Markers [42]. Die Bestimmung von Serum microRNA (miRNA) Signaturen erscheint nicht nur bei diabetischen Populationen zukünftig eine entsprechende Option zu werden ([43, 44]; Abb. 2).

\section{Basisprophylaxe mit Vitamin D und Kalzium}

Die Kalzium- und Vitamin D Substitution ist sowohl eine eigenständige Therapiemöglichkeit der Osteoporose, als auch die absolut notwendige Basis jeder spezifischen Osteoporosetherapie.

Eine ausreichende Versorgung mit Vitamin D ist eine wichtige Voraussetzung für die Knochengesundheit. Eine 25-OH-Vitamin D Serumkonzentration $<20 \mathrm{ng} / \mathrm{ml}(50 \mathrm{nmol} / \mathrm{l})$ ist mit einem erhöhten Risiko für proximale Femurfrakturen und nichtvertebralen Frakturen verbunden.

Zur Therapie eingesetzt wird Cholecalciferol (Vitamin D3); $1 \mu \mathrm{g}$ Vitamin D3 entspricht 40 IE Vitamin D3. Die Einnahme soll mit den Mahlzeiten erfolgen, da dies die Resorption verbessert. Die Tagesdosis (z. B. 800 IE) kann auch als Wochenäquivalent gegeben werden (5600 IE einmal wöchentlich). Im Einzelfall kann bei Malabsorption eine parenterale (intramuskuläre) Gabe von 100.000 IE Cholecalciferol notwendig sein. Die Gabe der aktiven Form von Vitamin D - Calcitriol (1,25-Dihydroxycholecalciferol) - ist nur bei schwerer Niereninsuffizienz indiziert.

Eine ausreichende Kalziumzufuhr ist primär über die Nahrung sicherzustellen. Patientinnen und Patienten mit Osteoporose (mit und ohne spezifischer Osteoporosetherapie) sollen daher täglich $1000 \mathrm{mg} \mathrm{Kal-}$ zium aufnehmen, vorzugsweise über die Nahrung. Ist dies nicht möglich, sind Kalziumsupplemente erforderlich. Pro Einnahme wird eine Dosis von maximal 500 mg Kalziumsupplement empfohlen [25].

\section{Spezifische Osteoporosetherapie bei Diabetes}

Keine einzige randomisierte Studie hatte bisher als Endpunkt die Wirksamkeit einer spezifischen Osteoporosetherapie bei Patienten mit T2DM. Daher basieren die Empfehlungen für das Management von Patienten mit Diabetes und einem erhöhten Frakturrisiko auf empirischen Daten und der klinischen Erfahrung. Die klinische Evidenz in Bezug auf die Effizienz einer antiresorptiven oder anabolen Osteoporosetherapie bei gleichzeitigem Diabetes beruht daher auf post hoc Analysen von Subgruppen in großen randomisierten Osteoporose Studien und auch einer kleinen Anzahl von Observationsstudien [45].

Grundsätzlich sind sämtliche Medikamente zur Behandlung der Osteoporose auch bei Patientinnen und Patienten mit einem manifesten Diabetes möglich und zugelassen. Da sowohl der Diabetes mellitus als auch die Osteoporose eine chronische Erkrankung mit einem dauerhaft erhöhten Risiko für sekundäre Komplikationen sind, ist die Indikation für eine langfristige Behandlung indiziert.

\section{Bisphosphonate}

Bisphosphonate (Alendronat, Risedronat, Ibandronat, Zoledronat) sind potente Inhibitoren der Knochenresorption. Sie werden an metabolisch aktiven Umbaueinheiten im Knochen abgelagert und bewirken eine Apoptose von Osteoklasten. Die Resorptionsaktivität wird im Gesamtskelett deutlich gedämpft und das Frakturrisiko reduziert.

Oral werden Bisphosphonate nur in geringem Ausmaß (maximal 3\%) resorbiert; die Einnahme erfolgt stets nüchtern in ausreichendem Abstand zur Nahrungsaufnahme, mit ausreichend Wasser und in aufrechter Körperhaltung, um Irritationen der Ösophagusschleimhaut $\mathrm{zu}$ vermeiden.

Bei intravenöser Bisphosphonatgabe kann, überwiegend bei erstmaliger Verabreichung, eine sogenannte „Akutphasereaktion“ - im Wesentlichen ein grippeähnliches Zustandsbild mit Fieber und Muskelschmerzen - auftreten, die in der Regel innerhalb von $36 \mathrm{~h}$ nach intravenöser Gabe beginnt und dann 24-48 h anhält.

Bei allen Bisphosphonaten stellen die Hypokalzämie, eine erhebliche Nierenfunktionseinschränkung oder eine Gravidität eine Kontraindikation dar.

Bisphosphonate haben eine lange Verweildauer im Knochen. Residuale Wirkungen auf den Knochenstoffwechsel lassen sich auch nach Beendigung der Bisphosphonattherapie nachweisen. Das Auftreten von atypischen Femurfrakturen ist sehr selten, scheint aber unter einer Langzeitgabe mit Bisphosphonaten zuzunehmen. Kiefernekrosen sind bei dieser für Os- 
teoporose zugelassenen Therapie eine mutmaßlich seltene Nebenwirkung. Eine Kontrolle des Zahnstatus ist allerdings vor Therapiebeginn empfehlenswert.

Es gibt keine durch Frakturdaten validierten individuellen Entscheidungskriterien für die Wiederaufnahme einer Therapie nach einer Therapiepause oder einen weiteren Therapieverzicht in Abhängigkeit von Veränderungen der BMD, der Knochenumbaumarker oder anderer messtechnischer oder klinischer Kriterien. Datenbankanalysen geben allerdings Hinweise auf einen Wiederanstieg des Knochenbruchrisikos nach Absetzen einer Bisphosphonattherapie [25].

\section{Denosumab}

Denosumab ist ein monoklonaler Antikörper gegen RANKL, der die Reifung und Aktivierung der Osteoklasten hemmt. Es wird alle sechs Monate subkutan verabreicht und wird nicht renal eliminiert.

Bei der Behandlung der postmenopausalen Osteoporose ist eine Reduktion von vertebralen und nichtvertebralen Frakturen inklusive proximaler Femurfrakturen in Studien bis zu 10 Jahre nachgewiesen. Die Wirkung ist unabhängig von einer eventuellen Vorbehandlung mit Bisphosphonaten [46]. Die Behandlungsdauer ist unklar. Nach Absetzen von Denosumab scheint es im Gegensatz zu den Bisphosphonaten zu einem raschen Anstieg des Knochenumbaus und in weiterer Folge $\mathrm{zu}$ einer Abnahme der Knochenmineraldichte $\mathrm{zu}$ kommen. Kiefernekrosen und atypische Femurfrakturen sind bei dieser für Osteoporose zugelassenen Therapie eine mutmaßlich sehr seltene Nebenwirkung [25].

\section{Raloxifen}

Raloxifen ist ein selektiver Östrogenrezeptor-Modulator (SERM), der die Knochenresorption hemmt und das Frakturrisiko für vertebrale Frakturen reduziert (nicht für nicht-vertebrale Frakturen und proximale Femurfrakturen). Raloxifen ist zugelassen für die Prävention und für die Therapie der Osteoporose bei postmenopausalen Frauen.

Ein bedeutender zusätzlicher Effekt ist die Reduktion des relativen Risikos eines invasiven (Östrogenrezeptor-positiven) Mammakarzinoms um $79 \%$. Eine unerwünschte Nebenwirkung ist die Erhöhung des thromboembolischen Risikos [25].

\section{Teriparatid}

Teriparatid, ein aminoterminales Fragment des Parathormons, wird einmal täglich subkutan über 24 Monate angewandt. Der osteoanabole Effekt beruht auf einer Beschleunigung der Reifung und Stimulierung von Osteoblasten.

Im Anschluss an die anabole Reaktion des Knochens kommt es nach Beendigung der TeriparatidTherapie wiederum $\mathrm{zu}$ einem gesteigerten Kno- chenabbau, weshalb eine sofortige Anschlussbehandlung mit einem Antiresorptivum (Bisphosphonat, Denosumab, SERM) unbedingt notwendig ist [25].

\section{Neue/zukünftige Osteoporose Medikamente}

Romosozumab, ein Anti-Sclerostin Antikörper, verbessert die BMD und die Knochenstärke im diabetischen Rattenmodell. Studiendaten bei postmenopausalen Frauen mit einem erhöhten Knochenbruchrisiko zeigen eine außergewöhnlich starke Zunahme der BMD bei monatlicher Applikation. Daher könnte dieser Antikörper zukünftig eine neue Behandlungsoption auch bei T2DM in der entsprechenden Indikation darstellen [47, 48].

\section{Management einer erhöhten Knochenfragilität bei Diabetes}

Die Kriterien für den Beginn einer osteologischen Therapie bei Diabetes basieren entweder auf einer prävalenten Fragilitätsfraktur (unabhängig von der BMD) und/oder auf einer verminderten BMD. Das diagnostische Kriterium der Osteoporose in der DXAMessung ( $\mathrm{T}$-score $<2,5$ ) ist nicht mit der individuellen Therapieschwelle gleich zu setzen $[25,49]$.

Die wichtigste Entscheidungshilfe für den Beginn einer Therapie ist auch beim Patienten mit Diabetes eine prävalente Fragilitätsfraktur. Das Ziel ist jedoch, die Patienten vor der ersten niedrig-traumatischen Fraktur zu schützen.

\section{BMD Interventionsschwelle}

Bei Patienten mit einem manifesten T2DM unterschätzt die DXA-Messung das individuelle Frakturrisiko. Aktuell wird daher bei diesen Patienten eine Anhebung der Interventionsschwelle auf einen T-score von -2,0 an der Lendenwirbelsäule (kumulativ L1-L4) oder an der Hüfte (Schenkelhals bzw. gesamte Hüfte) empfohlen, um der DXA-basierten Unterschätzung der Knochenfragilität entgegen zu wirken (Abb. 3). Diese Anhebung ist jedoch nur in westlichen Populationen zu empfehlen. Patienten aus Asien oder dem Nahen/Mittleren Osten haben bei Alters- und Geschlechts-adjustierter BMD niedrigere Frakturraten, die sich auch bei manifestem Diabetes auswirken.

Patienten mit einem ausgeprägten Verlust an BMD in zwei konsekutiven Messungen $(=>5 \%$ in zwei Jahren) sollten schon bei Werten nahe der Interventionsschwelle prophylaktisch behandelt werden.

\section{$F R A X^{\circledR}$}

FRAX, das WHO zertifizierte Fracture Risk Assessment Tool, implementiert nationale Frakturdaten und besteht aus 12 dichotomisierten Fragen (die 12. Frage zu BMD ist optional) [50]. Dem FRAX liegen klinische Risikofaktoren (clinical risk factor, CRF) zugrunde, die 
T2DM und Osteoporose

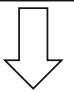

\section{Modifiktion des Lebensstils}

- Moderate Gewichtsreduktion

- Mediterrane Diät

- Nüsse/Keimlinge

- Vitamin D \pm Kalzium
- Alkoholkonsum reduzieren

- Rauchen beenden
- Gehen ( $\geq 150 \mathrm{~min} /$ Woche)

- Progressives

Widerstandstraining

\begin{tabular}{|c|}
\hline Behandlung des T2DM ${ }^{\mathrm{a}}$ \\
\hline $\begin{array}{l}\quad \text { Empfohlen }{ }^{\mathrm{a}} \\
\text { - Metformin } \\
\text { - GLP1-RA }\end{array}$ \\
\hline $\begin{array}{l}\text { Neutrala } \\
\text { - Empagliflozin, } \\
\text { Dapagliflozin } \\
\text { - DPP-4 Inhibitoren }\end{array}$ \\
\hline $\begin{array}{l}\quad \text { Kritisch } \\
\text { - TZDs } \\
\text { - Canagliflozin } \\
\text { - Insulin } \\
\text { - Sulfonylharnstoffe }\end{array}$ \\
\hline
\end{tabular}

Abb. 3 Strategien zur Behandlung von T2DM aus diabetologischer und osteologischer Sicht. (DPP4 Dipeptidyl Peptidase-4 Inhibitor, GLP-1 Glucagon-like peptide-1 Analoga, TZD Tiazolidindione, SGLT2 Natrium-Glucose Cotransporter 2 - Canagliflozin, CKD-MBD chronic kidney disease - metabolic bone disease, T2DM Diabetes mellitus Typ 2, Vitamin $D$

in randomisierten Studien ein erhöhtes individuelles Risiko für Fragilitätsfrakturen sind. FRAX berechnet zwei Werte: (a) eine 10-Jahreswahrscheinlichkeit für alle osteoporotischen Frakturen und (b) eine 10-Jahreswahrscheinlichkeit für eine osteoporotische Hüftfraktur. Entsprechend der Österreichischen Leitlinie zur Behandlung der Osteoporose wird ab einem Risiko von (a) $\geq 20 \%$ bzw. (b) $\geq 5 \%$ prophylaktisch eine knochenspezifische Therapie empfohlen.

Diabetes per se ist im FRAX kein eigener CRF, daher unterschätzt auch dieses diagnostische System die Frakturwahrscheinlichkeit bei einem manifesten Diabetes. Diabetes per se ist allerdings ein starker Risikofaktor für eine osteoporotische Fraktur, auch nach Korrektur aller CRFs und BMD [51]. FRAX bietet auch die Möglichkeit, die BMD-Werte mittels TBS-Korrek-
25-OH Vitamin D3 Cholecalciferol, GLP1-RA Glucagon-like peptide-1-Rezeptoragonisten, ÖDG LL Leitlinie der Österreichischen Diabetes Gesellschaft. "Unter Berücksichtigung weiterer klinischer Merkmale entsprechend der ÖDG-LL zur Antihyperglykämischen Therapie bei T2DM)

tur zu rechnen. Vor allem bei T2DM führt dies zu einer Verbesserung der Vorhersagewahrscheinlichkeit.

Berechnungen mit dem FRAX Rechner haben gezeigt, dass es weitere Korrekturmöglichkeiten gibt, um sich bei T2DM unter Verwendung dieses Risikorechners dem individuellen Frakturrisiko zu nähern. Eine Möglichkeit ist (a) die Erhöhung des Patientenalters um 10 Jahre, da der Risikofaktor Diabetes bei dieser Korrektur in etwa dem Risikofaktor Alter (als etablierter CRF im FRAX) entspricht. Die andere Möglichkeit ist (b) die Verminderung des gemessenen T-cores am Schenkelhals um 0,5 Standardabweichungen zu verringern (z.B. T-core $-2,4$ statt den tatsächlich gemessenen -1,9). Eine weitere Möglichkeit wäre es, (c) Rheumatoide Arthritis als CRF zu nehmen. Der Untersucher sollte sich für eine der drei Möglichkeiten 
Tab. 3 Risikofaktoren für Frakturen bei Patienten mit Diabetes

\begin{tabular}{l|l}
$\begin{array}{l}\text { Allgemeine Risikofaktoren } \\
\text { Krankheits-spezifische Risikofaktoren }\end{array}$ & $\begin{array}{l}\text { FRAX CRF: Alter, Geschlecht, prävalente Fragilitätsfrakturen, Hüftfraktur der Mutter oder des Vaters, gegen- } \\
\text { wärtiges Rauchen, Alkohol ( } \geq 3 \text { Einheiten/Tag), Glukokortikoide, Rheumatoide Arthritis, BMD Schenkelhals }\end{array}$ \\
\hline \begin{tabular}{l} 
Dauer des Diabetes $>5$ Jahre \\
\hline Antidiabetogene Medikamente: Insulin, TZDs, Canagliflozin, Sulfonylharnstoffe
\end{tabular} & $\begin{array}{l}\text { HbA1C }>7 \% \\
\text { Mikrovaskuläre Komplikationen: periphere und autonome Neuropathie, Retinopathie, Nephropathie }\end{array}$ \\
\hline FRAXFracture Risk Assessment Tool der WH0, CRFclinical risk factors, TZD Thiazolidindione, BMD „bone mineral density“
\end{tabular}

entscheiden, jedoch nicht alle drei Optionen verwenden.

Nach aktueller Datenlage bieten diese drei Optionen trotz aller methodischen Limitationen derzeit die beste Lösung, sich dem tatsächlichen individuellen Frakturrisiko bei T2DM zu nähern. Die größte Trefferwahrscheinlichkeit bietet die Verwendung des CRF Rheumatoide Arthritis und wird daher derzeit auch von der Task Force Diabetes der International Osteoporosis Foundation empfohlen ([45]; Tab. 3).

\section{Zusammenfassung}

Patienten mit Diabetes haben ein erhöhtes Risiko für Fragilitätsfrakturen. Die Pathophysiologie ist unklar und vermutlich multifaktoriell.

Longitudinale Studien haben den Nachweis erbracht, dass FRAX und BMD mittels DXA (T-score) Messungen und einem eventuell vorhandenen TBS das individuelle Frakturrisiko vorhersagen können. Hierfür muss allerdings eine Adjustierung vorgenommen werden, um das Risiko nicht zu unterschätzen.

Bei der Wahl der antihyperglykämischen Therapie sollen Substanzen mit nachgewiesenem negativem Effekt auf den Knochen nicht zum Einsatz kommen. Bei Vorliegen einer Fragilitätsfraktur ist auf jeden Fall unabhängig von allen vorliegenden Befunden - eine langfristige spezifische osteologische Therapie indiziert.

Es gibt zurzeit keine Daten aus einem rein diabetischen Kollektiv, weshalb der hier empfohlene Algorithmus einen Konsens einer internationalen Expertengruppe (somit Evidenzgrad D) darstellt.

Demnach sollen Patienten mit Diabetes mellitus und einem erhöhten Frakturrisiko wie Patienten ohne Diabetes behandelt werden.

Auf einen optimalen Vitamin D Spiegel sowie eine ausreichende Kalziumaufnahme (vorzugsweise durch die Nahrung) sollte geachtet werden.

Zur Prävention von Fragilitätsfrakturen sind antiresorptive Medikamente die erste Wahl, entsprechend der nationalen Erstattungskriterien auch anabole Medikamente. Das Therapiemonitoring soll im Einklang mit der nationalen Osteoporose Leitlinie erfolgen [25].

Funding Open access funding provided by Medical University of Vienna.

Interessenkonflikt C. Muschitz hat von folgenden Firmen, die auch fördernde Mitglieder der ÖGKM (Österr. Gesell- schaft für Knochen- und Mineralstoffwechsel) und der ÖDG sind, Honorare für Vorträge und Beratung sowie wissenschaftliche Grants erhalten: Amgen Österreich; Eli Lilly Österreich, Novartis Pharma Österreich. Es besteht kein Konflikt mit der Erstellung dieses Manuskripts. A. Kautzky-Willer hat von folgenden Firmen, die teils auch fördernde Mitglieder der ÖDG sind, Honorare für Vorträge und Beratung erhalten: AstraZeneca, Amgen, Boehringer Ingelheim, Eli Lilly, Merck Sharp \& Dohme, Novartis, Novo Nordisk, Roche, sanofi-aventis, Servier, Takeda. M. Rauner hat von folgender Firma, die auch förderndes Mitglieder der ÖGKM ist, Honorare für Vorträge und Beratung erhalten: Amgen. Es besteht kein Konflikt mit der Erstellung dieses Manuskripts. Y. Winhöfer-Stöckl hat von folgenden Firmen, die auch teils auch fördernde Mitglieder der ÖDG sind, Honorare für Vorträge und Beratung erhalten: Amgen, Astra Zeneca, Eli Lilly, Novartis, Sanofi. J. Haschka hat keine Honorare von Firmen für Vorträge oder Beratung erhalten.

Open Access Dieser Artikel wird unter der Creative Commons Namensnennung 4.0 International Lizenz (http:// creativecommons.org/licenses/by/4.0/deed.de) veröffentlicht, welche die Nutzung, Vervielfältigung, Bearbeitung, Verbreitung und Wiedergabe in jeglichem Medium und Format erlaubt, sofern Sie den/die ursprünglichen Autor(en) und die Quelle ordnungsgemäß nennen, einen Link zur Creative Commons Lizenz beifügen und angeben, ob Änderungen vorgenommen wurden.

Hinweis des Verlags Der Verlag bleibt in Hinblick auf geografische Zuordnungen und Gebietsbezeichnungen in veröffentlichten Karten und Institutsadressen neutral.

\section{Literatur}

1. Shah VN, Shah CS, Snell-Bergeon JK. Type 1 diabetes and risk of fracture: meta-analysis and review of the literature. Diabet Med. 2015;32(9):1134-42.

2. Weber DR, Haynes K, Leonard MB, Willi SM, Denburg MR. Type 1 diabetes is associated with an increased risk of fracture across the life span: a population-based cohort study using The Health Improvement Network (THIN). Diabetes Care. 2015;38(10):1913-20.

3. Holmberg AH, Johnell O, Nilsson PM, Nilsson J, Berglund G, Akesson K. Risk factors for fragility fracture in middle age. A prospective population-based study of 33,000 men and women. Osteoporos Int. 2006;17(7):1065-77.

4. Chamberlain JJ, Herman WH, Leal S, Rhinehart AS, Shubrook JH, Skolnik N, et al. Pharmacologic therapy for type 2 diabetes: synopsis of the 2017 American Diabetes Association standards of medical care in diabetes. Ann Intern Med. 2017;166(8):572-8.

5. Leslie WD, Morin SN, Lix LM, Majumdar SR. Does diabetes modify the effect of FRAX risk factors for predict- 
ing major osteoporotic and hip fracture? Osteoporos Int. 2014;25(12):2817-24.

6. Majumdar SR, Leslie WD, Lix LM, Morin SN, Johansson H, Oden A, et al. Longer duration of diabetes strongly impacts fracture risk assessment: the manitoba BMD cohort. J Clin Endocrinol Metab. 2016;101(11):4489-96.

7. Li CI, Liu CS, Lin WY, Meng NH, Chen CC, Yang SY, et al. Glycated hemoglobin level and risk of hip fracture in older people with type 2 diabetes: a competing risk analysis of Taiwan diabetes cohort study. J Bone Miner Res. 2015;30(7):1338-46.

8. Shanbhogue VV, Hansen S, Frost M, Jorgensen NR, Hermann AP, Henriksen JE, et al. Compromised cortical bone compartment in type 2 diabetes mellitus patients with microvascular disease. Eur JEndocrinol. 2016;174(2):115-24.

9. Napoli N, ShahK, Waters DL, SinacoreDR, Qualls C, Villareal DT. Effect of weight loss, exercise, or both on cognition and quality of life in obese older adults. Am J Clin Nutr. 2014;100(1):189-98.

10. Hurskainen AR, Virtanen JK, Tuomainen TP, Nurmi T, Voutilainen S. Association of serum 25-hydroxyvitamin D with type 2 diabetes and markers of insulin resistance in a general older population in Finland. Diabetes Metab Res Rev. 2012;28(5):418-23.

11. Bolland MJ, Grey A, Avenell A. Effects of vitamin D supplementation on musculoskeletal health: a systematic review, meta-analysis, and trial sequential analysis. Lancet Diabetes Endocrinol. 2018;6(11):847-58.

12. Conway BN, Long DM, Figaro MK, May ME. Glycemic control and fracture risk in elderly patients with diabetes. Diabetes Res Clin Pract. 2016;115:47-53.

13. InzucchiSE, BergenstalRM, BuseJB, DiamantM, Ferrannini E, Nauck M, et al. Management of hyperglycemia in type 2 diabetes, 2015: a patient-centered approach: update to a position statement of the American Diabetes Association and the European Association for the Study of Diabetes. Diabetes Care. 2015;38(1):140-9.

14. Napoli N, Chandran M, Pierroz DD, Abrahamsen B, Schwartz AV, Ferrari SL, et al. Mechanisms of diabetes mellitus-induced bone fragility. Nat Rev Endocrinol. 2017;13(4):208-19.

15. Smieszek A, Tomaszewski KA, Kornicka K, Marycz K. Metformin promotes osteogenic differentiation of adiposederived stromal cells and exerts pro-osteogenic effect stimulating bone regeneration. J Clin Med. 2018;7(12):482.

16. Liu Q, Xu X, Yang Z, Liu Y, Wu X, Huang Z, et al. Metformin alleviates the boneloss induced byketogenic diet: an in vivo study in mice. CalcifTissue Int. 2019;104(1):59-69.

17. Vianna AGD, Sanches CP, Barreto FC. Review article: effects of type 2 diabetes therapies on bone metabolism. Diabetol Metab Syndr. 2017;9:75.

18. Gilbert MP, Marre M, Holst JJ, Garber A, Baeres FM, Thomsen $\mathrm{H}$, et al. Comparison of the long-term effects of liraglutide and glimepiride monotherapy on bone mineral density in patients with type 2 diabetes. Endocr Pract. 2016;22(4):406-11.

19. Dormuth CR, Carney G, Carleton B, Bassett K, Wright JM. Thiazolidinediones and fractures in men and women. Arch Intern Med. 2009;169(15):1395-402.

20. Paschou SA, Dede AD, Anagnostis PG, Vryonidou A, Morganstein D, Goulis DG. Type 2 diabetes and osteoporosis: a guide to optimal management. J Clin Endocrinol Metab. 2017;102(10):3621-34.

21. Viscoli CM, Inzucchi SE, Young LH, Insogna KL, Conwit R, Furie KL, et al. Pioglitazone and risk for bone fracture: safety data from a randomized clinical trial. J Clin Endocrinol Metab.2017;102(3):914-22.
22. Wallander M, Axelsson KF, Nilsson AG, Lundh D, Lorentzon M. Type 2 diabetes and risk of hip fractures and non-skeletal fall injuries in the elderly: a study from the Fractures and Fall Injuries in the Elderly Cohort (FRAILCO). J Bone Miner Res. 2017;32(3):449-60.

23. Palermo A, D’Onofrio L, Eastell R, Schwartz AV, Pozzilli P, Napoli N. Oral anti-diabetic drugs and fracture risk, cut to the bone: safe or dangerous? A narrative review. Osteoporos Int. 2015;26(8):2073-89.

24. Ruanpeng D, Ungprasert P, Sangtian J, Harindhanavudhi T. Sodium-glucose cotransporter 2 (SGLT2) inhibitors and fracture risk in patients with type 2 diabetes mellitus: a meta-analysis. Diabetes Metab Res Rev. 2017;33(6):e2903.

25. Initiative „Arznei \& Vernunft“ - ein gemeinsames Projekt von Hauptverband der österreichischen Sozialversicherungsträger, Pharmig, Österreichischer Ärztekammer und Österreichischer Apothekerkammer; www. arzneiundvernunft.at

26. Hough FS, Pierroz DD, Cooper C, Ferrari SL, Bone IC, DiabetesWorking G. Mechanisms in endocrinology: mechanisms and evaluation of bone fragility in type 1 diabetes mellitus. EurJEndocrinol.2016;174(4):R127-R38.

27. Ma L, Oei L, Jiang L, Estrada K, Chen H, Wang Z, et al. Association between bone mineral density and type 2 diabetes mellitus: a meta-analysis of observational studies. EurJEpidemiol.2012;27(5):319-32.

28. Schacter GI, Leslie WD. DXA-based measurements in diabetes: Can they predict fracture risk? Calcif Tissue Int. 2017;100(2):150-64.

29. LeslieWD, Morin SN, Majumdar SR, Lix LM. Effects of obesity and diabetes on rate of bone density loss. Osteoporos Int. 2018;29(1):61-7.

30. Muschitz C, Kocijan R, Haschka J, Pahr D, Kaider A, Pietschmann $\mathrm{P}$, et al. TBS reflects trabecular microarchitecture in premenopausal women and men with idiopathic osteoporosis and low-traumatic fractures. Bone. 2015;79:259-66.

31. McCloskey EV, Oden A, Harvey NC, Leslie WD, Hans D, Johansson $\mathrm{H}$, et al. A meta-analysis of trabecular bone score in fracture risk prediction and its relationship to FRAX. JBone Miner Res. 2016;31(5):940-8.

32. Leslie WD, Aubry-Rozier B, Lamy O, Hans D, Manitoba Bone Density P.TBS (trabecular bone score) and diabetes-related fracture risk. JClin Endocrinol Metab. 2013;98(2):602-9.

33. Yamaguchi T, Yamamoto M, Kanazawa I, Yamauchi M, Yano S, Tanaka N, et al. Quantitative ultrasound and vertebral fractures in patients with type 2 diabetes. J Bone Miner Metab. 2011;29(5):626-32.

34. Nilsson AG, Sundh D, Johansson L, Nilsson M, Mellstrom $\mathrm{D}$, Rudang $\mathrm{R}$, et al. Type 2 diabetes mellitus is associated with better bone microarchitecture but lower bone material strength and poorer physical function in elderly women: a population-based study. J Bone Miner Res. 2017;32(5):1062-71.

35. Patsch JM, Rasul S, Huber FA, Leitner K, Thomas A, Kocijan $\mathrm{R}$, et al. Similarities in trabecular hypertrophy with sitespecific differences in cortical morphology between men and women with type 2 diabetes mellitus. PLoS ONE. 2017;12(4):e174664.

36. Ferrari S. Diabetes and bone. Calcif Tissue Int. 2017;100(2): 107-8.

37. Manavalan JS, Cremers S, Dempster DW, Zhou H, Dworakowski E, Kode A, et al. Circulating osteogenic precursor cells in type 2 diabetes mellitus. J Clin Endocrinol Metab. 2012;97(9):3240-50.

38. Tanaka S, Kuroda T, Saito M, Shiraki M. Urinary pentosidine improves risk classification using fracture risk assess- 
ment tools for postmenopausal women. J Bone Miner Res. 2011;26(11):2778-84.

39. Ardawi MS, Akhbar DH, Alshaikh A, Ahmed MM, Qari $\mathrm{MH}$, Rouzi AA, et al. Increased serum sclerostin and decreased serum IGF-1 are associated with vertebral fractures among postmenopausal women with type-2 diabetes. Bone. 2013;56(2):355-62.

40. Starup-Linde J, Lykkeboe S, Gregersen S, Hauge EM, Langdahl BL, Handberg A, et al. Bone structure and predictors of fracture in type 1 and type 2 diabetes. J Clin Endocrinol Metab. 2016;101(3):928-36.

41. Morales-Santana S, Garcia-Fontana B, Garcia-Martin A, Rozas-Moreno P, Garcia-Salcedo JA, Reyes-Garcia R, et al. Atherosclerotic disease in type 2 diabetes is associated with an increase in sclerostin levels. Diabetes Care. 2013;36(6):1667-74.

42. Pepe J, Bonnet N, Herrmann FR, Biver E, Rizzoli R, Chevalley $\mathrm{T}$, et al. Interaction between LRP5 and periostin gene polymorphisms on serum periostin levels and cortical bone microstructure. Osteoporos Int. 2018;29(2):339-46.

43. Heilmeier U, Hackl M, Skalicky S, Weilner S, Schroeder F, Vierlinger K, et al. Serum miRNA signatures are indicative of skeletal fractures in postmenopausal women with and without type 2 diabetes and influence osteogenic and adipogenic differentiation of adipose tissue-derived mesenchymal stem cells in vitro. J Bone Miner Res. 2016;31(12):2173-92.

44. Feichtinger X, Muschitz C, Heimel P, Baierl A, FahrleitnerPammer A, Redl H, et al. Bone-related circulating microRNAs miR-29b-3p, miR-550a-3p, and miR-324-3p and their association to bone microstructure and histomorphometry. Sci Rep. 2018;8(1):4867.

45. Ferrari SL, Abrahamsen B, Napoli N, Akesson K, Chandran $\mathrm{M}$, Eastell R, et al. Diagnosis and management of bone fragility in diabetes: an emerging challenge. Osteoporos Int. 2018;29(12):2585-96.

46. Tsourdi E, Makras P, Rachner TD, Polyzos S, Rauner M, Mandanas S, et al. Denosumab effects on bone density and turnover in postmenopausal women with low bone mass with or without previous treatment. Bone. 2018;120:44-9.

47. Hamann C, Rauner M, Hohna Y, Bernhardt R, Mettelsiefen J, Goettsch C, et al. Sclerostin antibody treatment improves bone mass, bone strength, and bone defect regeneration in rats with type 2 diabetes mellitus. J Bone Miner Res. 2013;28(3):627-38.

48. Saag KG, Petersen J, Brandi ML, Karaplis AC, Lorentzon M, Thomas T, et al. Romosozumab or alendronate for fracture prevention in women with osteoporosis. N Engl J Med. 2017;377(15):1417-27.

49. World Health Organization. Assessment of fracture risk and its application to screening for postmenopausal osteoporosis : report of a WHO study group [meeting held in Rome from 22 to 25 June 1992]. Geneva : World Health Organization. 1994. http://www.who.int/iris/handle/10665/39142.

50. FRAX. www.sheffield.ac.uk/FRAX

51. Giangregorio LM, Leslie WD, Lix LM, Johansson H, Oden A, McCloskey E, et al. FRAX underestimates fracture risk in patients with diabetes. J Bone Miner Res. 2012;27(2):301-8. 